\title{
Die stationäre universitäre psychosomatische Behandlung gehört ins Inselspital!
}

Alexander Minzer

Präsident SAPPM

Korrespondenz:

Dr. med. Alexander Minzer

Allgemeine Innere Medizin, Mitglied FMH

Psychosomatische und

Psychosoziale Medizin SAPPM

Breitenstrasse 15

CH-4852 Rothrist

alexander.minzer[at]hin.ch
In der heutigen Zeit ist eine drastische Zunahme von Stressfolgekrankheiten und Krankheiten aus dem gemischt somatisch-psychischen Formenkreis zu verzeichnen. Trotz dieser Tatsachen hat die Klinikleitung des Inselspitals Bern vorgesehen, die einzige stationär universitäre Abteilung für Psychosomatische Medizin in der Schweiz, mit ihren 28 Betten, die permanent ausgelastet ist und für die eine Warteliste besteht, zu schliessen. Das multidisziplinäre, hochspezialisierte Team, bestehend aus 50 Mitarbeitern, soll entlassen werden. Die Schweizerische Akademie für Psychosomatische und Psychosoziale Medizin kann diese Massnahme in keiner Weise gutheissen und fordert ein Umdenken sowie das Aufheben dieses Entscheides.

Per 1. oder 2. Quartal 2014 soll im Inselspital Bern die einzige universitäre Abteilung für stationäre Psychosomatik geschlossen werden. Ende September stand es im «Bund» [1]. Die Schweizerische Akademie für Psychosomatische und Psychosoziale Medizin SAPPM sieht in diesem mit wirtschaftlichen Überlegungen begründeten Entscheid einen Rückschritt in der medizinischen, speziell der universitären Versorgung der Schweiz!

Dafür wird auf Transplantations- und Herzchirurgie gesetzt. Sind dies die erwarteten Folgen der Einführung des Swiss-DRG? Oder stehen nicht auch Prestige-Überlegungen dahinter? Ist es nicht einfach werbewirksamer, Herzen zu transplantieren als chronisch kranke Patienten zu behandeln?

Die Klinik für Psychosomatische Medizin am Inselspital stellt eine traditionsreiche und erfolgreich etablierte Abteilung der Universitätsklinik für Allgemeine Innere Medizin dar. Die 1978 von Professor Rolf Adler ins Leben gerufene, einzige universitäre Klinik für stationäre Psychosomatische Medizin geniesst schweizweit und im Ausland einen ausgezeichneten Ruf. Im Laufe der Jahrzehnte wurde die Abteilung stetig vergrössert. Professor Rolf Adler war als Chefarzt der Psychosomatik zwischenzeitlich Leiter des Departements für Innere Medizin am Inselspital, was die Bedeutung der Klinik mehr als unterstreicht. Seit über fünfunddreissig Jahren wurde, aktuell nun unter Professor Roland von Känel, an der Klinik für Psychosomatik geforscht und herausragende Arbeit geleistet.

Körperliche Erkrankungen und Beschwerden, mitverursacht oder verstärkt durch psychische oder psychosoziale Faktoren, zählen heute zu den häufigsten Erkrankungen und bedingen - einerseits durch Arbeitsunfähigkeit und andererseits wiederholte, nicht fachgerechte Diagnostik und inadäquate Therapien - hohe volkswirtschaftliche Kosten. Der Bereich Psychosomatische Medizin an der Universitätsklinik für Allgemeine Innere Medizin am Inselspital Bern berücksichtigt die körperlichen, seelischen und sozialen Aspekte von Krankheit und Gesundheit gleichwertig. Diese Arbeitsweise orientiert sich am biopsychosozialen Modell von Gesundheit und Krankheit und wird getragen durch die interdisziplinäre $\mathrm{Zu}$ sammenarbeit von universitärer Dienstleistung, Lehre und Forschung. Der hervorragende Ruf des Chefarztes Prof. Roland von Känel in internationalen wissenschaftlichen Fachkreisen unterstreicht den Stellenwert des Fachgebietes innerhalb der gesamten Medizin. Für die 28 stationären Behandlungsplätze besteht eine Warteliste.

Angesichts der dramatischen Zunahme von Stressfolgekrankheiten besteht ein vitales Interesse der Gesellschaft an der Erforschung, Prävention und Therapie dieser Störungen. Hochqualifizierte und erfahrene Spezialisten und Spezialistinnen - aus dem ärztlichen Bereich, der Psychologie, Pflege, Physiotherapie, Ergotherapie, Logopädie und Sozialberatung - arbeiten in diesem Bereich eng zusammen und stellen den Patienten mit seinen individuellen Bedürfnissen und Voraussetzungen in den Mittelpunkt.

Dieses Angebot steht Behandlungsbedürftigen aller Versicherungskategorien aus dem In- und Ausland offen. Aktuell stellt die Psychosomatik Lory ein schweizweit einzigartiges stationäres Therapiemodell dar, welches von Zuweisern und Patienten aus dem ganzen Land geschätzt und in Anspruch genommen wird. Häufig werden auch Patienten von anderen Kliniken des Inselspitals zur spezialisierten Behandlung auf die Lory-Abteilung verlegt.

Die Übernahme akut erkrankter Patienten ermöglicht nicht nur die kostengünstige und fachgerechte Therapie, sondern erlaubt unter Zusammenarbeit mit Angehörigen, Sozialversicherungen und Hausärzten die Planung der langfristigen ambulanten Versorgung sowie im Idealfall die berufliche Wiedereingliederung.

Umso unverständlicher erscheint der Beschluss der Spitalleitung, die stationäre Psychosomatik in der ersten Jahreshälfte 2014 zu schliessen. 


\section{Aktuelle}

Forumthemen

Diskutieren Sie mit! Im Forum präsentieren wir regelmässig brisante Themen aus Politik, Ökonomie und Wissenschaft, die das Schweizer Gesundheitswesen betreffen. Bringen Sie Ihre Meinung ein oder kommentieren Sie die Äusserungen Ihrer Kolleginnen und Kollegen. Das Forum finden Sie unter: www.saez.ch/forum/
Stichhaltige Argumente für eine solche Massnahme konnten bisher nicht geliefert werden, eine offizielle Stellungnahme der Spitalleitung steht bis heute aus, obschon einem Grossteil der 50 Mitarbeiter die Kündigung bereits zum 1. Dezember ausgesprochen werden soll. In Mangelberufen langjährig tätige, hochqualifizierte Fachkräfte gehen verloren. Hochspezialisierte, eingespielte Teams sollen aufgelöst und damit komplexe Kompetenzen vernichtet werden.

Angesichts dieser Perspektive reagieren nicht nur die betroffenen Mitarbeiter, sondern vor allem auch Fachverbände, zuweisende Ärzte und Patienten mit Unverständnis.

Für Menschen mit komplexen Schmerzerkrankungen, ausgeprägten Funktionsstörungen, schweren körperlichen oder psychiatrischen Begleiterkrankungen würde im Fall der Schliessung keine fachkompetente universitäre Therapieoption mehr zur Verfügung stehen.

Insbesondere aber würde auch den meist jungen Patientinnen mit schweren Mangelernährungszuständen im Rahmen einer Magersucht keine adäquate stationäre Behandlung mehr offenstehen. Da Patientinnen mit sehr niedrigem Gewicht häufig vital gefährdet sind und somit einer intensiven somatischen Betreuung und Behandlung bedürfen, stellt sich hier auch die Frage nach der ethischen Verantwortung der gesundheitspolitischen Entscheidungsträger.

Das Inselspital steht als Zentrum der Maximalversorgung in der Pflicht, alle Kranken aufzunehmen und fachgerecht zu behandeln. Als zuständige Therapeuten und Ärzte sehen wir eine berufsethische und humanitäre Verantwortung für diejenigen Patientengruppen, die auf unsere interdisziplinäre Fachkompetenz angewiesen sind.

Wirksamkeit und Kosteneffizienz psychosomatischer Komplextherapien sind seit langem bewiesen. Vor diesem Hintergrund wird die Kapazität an hochspezialisierten stationären Therapieangeboten in Deutschland und an schweizerischen Privatkliniken stetig ausgebaut. Es stellt einen Anachronismus und eine gesellschaftspolitische Fehlleistung dar, diese Fachkompetenzen ausgerechnet im universitären Milieu eliminieren zu wollen. In diesem Kontext muss auch darauf hingewiesen werden, dass alle(!)
Medizinstudenten der Universität Bern in Anamnesekursen und Kommunikationstrainings sowie spezifischen Vorlesungen an der Lory-Klinik psychosomatisches Grundverständnis erwerben. Auch hiermit leistet die Klinik für Psychosomatik einen unersetzlichen Beitrag zu den von Bevölkerung und Politik erwarteten Kompetenzen zukünftiger Ärztegenerationen.

Bereits seit Jahren wurde der Bereich Psychosomatik am Inselspital durch Bettenstreichungen bei gleichbleibender Leistungsanforderung unverhältnismässig strapaziert. Der Umgang mit den nun erneut betroffenen Mitarbeitern steht in irritierendem Widerspruch zu einem Menschlichkeit, Fairness und Qualität preisenden Leitbild des Inselspitals.

Der Versuch, ohne stichhaltige Sachargumente die stationäre Psychosomatik am Universitätsspital abzuschaffen, verlangt nach einer fachlichen, gesellschaftspolitischen und ethischen Diskussion. Nicht nur die «Sprechende Medizin», auch der Ruf des Inselspitals steht auf dem Spiel!

Aus Sicht der SAPPM macht es den Anschein, dass seit der Einführung von DRG, die Spitäler aus wirtschaftlichen Überlegungen zunehmend dazu gezwungen werden, sogenannte «nicht rentable» oder «unattraktive» medizinische Disziplinen aus den Kliniken zu verbannen. Zum einen, weil während eines stationären Aufenthalts nichts finanziell zu holen ist (niedrige Abgeltung über DRG), zum andern, weil gerade Patienten, die chronisch krank sind oder an psychosomatischen Krankheitsbildern leiden, sehr betreuungs- und personalintensiv sind. Und dies liesse sich bei fehlender tarifärer Abgeltung im DRG angeblich nicht mehr finanzieren. Sind dies aber nicht sehr kurzfristige Blickwinkel auf Kosten, die uns langfristig garantiert einholen und uns dann wesentlich teurer zu stehen kommen werden?

Somit soll nun eine hochspezialisierte Abteilung eines Universitätsspitals geschlossen werden. Dagegen gilt es anzukämpfen, mit allen zur Verfügung stehenden Mitteln!

Die Psychosomatik gehört in ein somatisches Umfeld und damit ins Inselspital!

1 Der Bund online vom 26.9.2013: «Die Psychosomatik ist dem Universitätsspital zu teuer.» 